\title{
Noninvasive evaluation of renal tissue oxygenation with blood oxygen level-dependent magnetic resonance imaging early after transplantation has a limited predictive value for the delayed graft function
}

\author{
Agata Sławińska ${ }^{1 B, D, E, F}, Z^{2}$ bigniew Serafin ${ }^{1 A, B, C, D, E, F, G,}$, Elżbieta Zawada ${ }^{1 A, B, D}$, Marcin Białecki ${ }^{1 A, B, D}$, \\ Katarzyna Wypych ${ }^{1 A, B, D, E}$, Aleksandra Woderska ${ }^{2 A, B}$, Maciej Słupski ${ }^{3 A, B}$, Zbigniew Włodarczyk ${ }^{2 A, B}$ \\ 'Department of Radiology and Diagnostic Imaging, Nicolaus Copernicus University, Collegium Medicum, Bydgoszcz, Poland \\ ${ }^{2}$ Department of General and Transplant Surgery, Nicolaus Copernicus University, Collegium Medicum, Bydgoszcz, Poland \\ ${ }^{3}$ Department of Hepatobiliary and General Surgery, Nicolaus Copernicus University, Collegium Medicum, Bydgoszcz, Poland
}

\section{Abstract}

\begin{abstract}
Purpose: The aim of this study was to evaluate the feasibility of renal oxygenation assessment using blood oxygen level-dependent (BOLD) magnetic resonance imaging (MRI) in the early period after kidney transplantation and to estimate its prognostic value for delayed graft function.

Material and methods: Examinations were performed in 50 subjects: 40 patients within a week after the kidney transplantation and 10 healthy controls, using T2*-weighted sequence. Measurements in transplant patients were correlated to basic laboratory parameters in the early period after transplantation and at follow-up.

Results: Examinations of seven patients $(18 \%)$ were rejected due to their poor technical quality. Mean R2* values in transplant recipients were lower than in controls $(11.6$ vs. $15.9 \mathrm{~Hz} ; p=0.0001)$. An $\mathrm{R} 2^{\star}$ value of $0.28 \mathrm{~Hz}$ was calculated as the minimal detectable change. There was no relation between $\mathrm{R} 2{ }^{*}$ values and laboratory parameters. However, patients eGFR $\geq 40 \mathrm{ml} / \mathrm{min} / 1.73 \mathrm{~m}^{2}$ presented higher R2* values than recipients eGFR $<40 \mathrm{ml} / \mathrm{min} / 1.73 \mathrm{~m}^{2}$ ( 12.0 vs. $11.1 \mathrm{~Hz} ; p=0.0189)$. In ROC analysis $\mathrm{R} 2^{*}$ of $\leq 11.7$ predicted an early reduced graft function with 0.82 sensitivity and $56 \%$ specificity (AUC $=0.708 ; p=0.024$ ) but was not useful for delayed graft function prediction $(p>0.7)$.

Conclusions: Evaluation of renal graft oxygenation using BOLD MRI is technically challenging in the early period after transplantation. An $2^{*}$ value of $0.28 \mathrm{~Hz}$ may in practice be considered as the minimal detectable change. The delayed graft function seems not to be dependent on early oxygenation values. Further, large-scale studies are necessary to confirm the latter observation.
\end{abstract}

Key words: renal graft oxygenation, BOLD MRI, blood oxygen level dependent (BOLD) magnetic resonance, kidney transplantation.

\section{Introduction}

Renal transplantation is the most effective therapeutic method for end-stage chronic kidney disease (CKD). Unfortunately, there are currently no good markers of early allograft injury, and a kidney biopsy is often required for diagnostic purposes [1]. However, the clinical utility of this approach is not clear. Biopsy also carries the risk of significant graft injury, and it cannot be performed in patients who take anticoagulants [2]. Therefore, current best practice involves vigilant monitoring of simple markers of allograft function, such as serum creatinine and pro-

Correspondence address:

Prof. Zbigniew Serafin, Department of Radiology and Diagnostic Imaging, Nicolaus Copernicus University, Collegium Medicum, 9 Skłodowskiej-Curie St.,

85-094 Bydgoszcz, Poland, e-mail: serafin@cm.umk.pl

Authors' contribution:

A Study design · B Data collection · C Statistical analysis · D Data interpretation · E Manuscript preparation · F Literature search · G Funds collection 


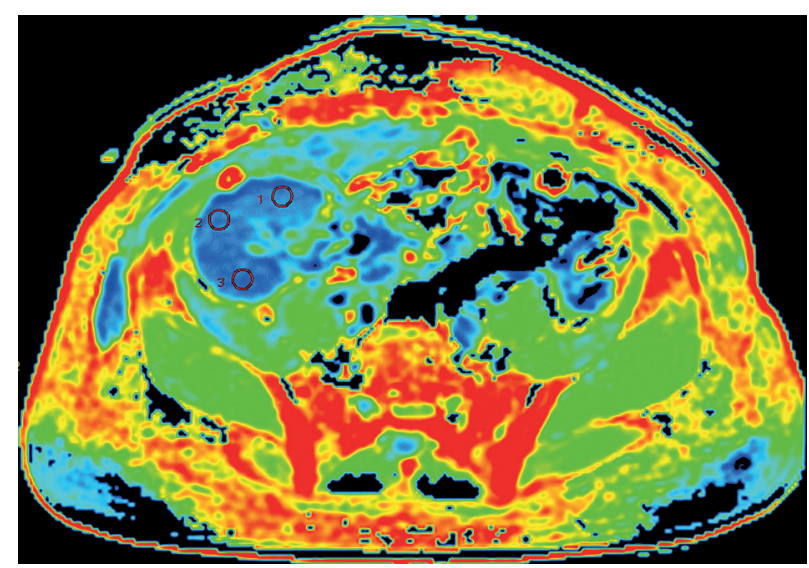

Figure 1. Example of ROls placement in a transplanted kidney on axial R2* map

teinuria. More complex and expensive approaches, such as monitoring of serum anti-HLA antibodies, remain unproven [3]. Thus, a non-invasive, fast, and specific tool that would detect acute allograft parenchymal pathologies is desirable [2].

Magnetic resonance imaging (MRI) could become a one-stop shop modality for transplanted kidney evaluation, as it is an established method for renal graft morphology and vasculature assessment $[4,5]$. Recently, several MRI techniques that evaluate renal graft function have been introduced [6-8]. For instance, blood oxygen level-dependent (BOLD) imaging can depict blood flow and parenchymal oxygen bioavailability $[9,10]$, which may be related to renal graft metabolism and thus can become a surrogate biomarker of graft survival.

BOLD MRI can be used for non-invasive estimation of renal parenchymal oxygen concentration. BOLD MRI is based on the paramagnetic effect of deoxyhaemoglobin for indirect depiction of renal oxygenation, whereby an increased oxygen consumption results in an increased deoxygenated haemoglobin concentration. This leads to decreased $\mathrm{T} 2{ }^{*}$ relaxation times on renal parenchyma images, which can be measured as the rate of spin dephasing $\left(\mathrm{R} 2^{\star}=1 / \mathrm{T} 2^{*}\right)[9]$. Thus, $\mathrm{R} 2^{\star}$ may be considered as an indicator of renal metabolism [10]. However, factors other than oxygenation, such as perfusion, haematocrit, or microstructure, can influence the deoxyhaemoglobin concentration and thus the $\mathrm{R} 2^{*}$ values.

It was shown that renal cortical oxygenation, measured with BOLD imaging, was reduced in patients with CKD, and cortical oxygenation level correlated with CKD severity [11]. BOLD imaging was also suggested as a method to diagnose early kidney graft failure [12-17]. To our knowledge, no study has assessed the role of renal cortex oxygenation measurement early after transplantation in the prediction of delayed renal graft function. Therefore, we aimed to assess the feasibility of BOLD imaging in the early period after kidney transplantation in order to establish $\mathrm{R} 2^{\star}$ measurement variability and to verify the value of $\mathrm{R} 2^{\star}$ in predicting delayed graft function.

\section{Material and methods}

We performed renal BOLD examinations in 40 kidney graft recipients (patients) and 10 healthy controls. Patients were included if their clinical state enabled MRI examination within the first week after transplantation. Controls with no history of kidney disease were included if their eGFR was $\geq 90 \mathrm{ml} / \mathrm{min} / 1.73 \mathrm{~m}^{2}$. Exclusion criteria were as follows: claustrophobia, metallic implants, dyspnoea, and peritoneal dialysis. Informed consent was obtained from all participants. The study was approved by our University Review Board.

MRI examinations were performed with a 1.5 -T scanner (GE Optima 450wGEM, General Electric Healthcare, Waukesha, USA). In patients, the examinations were performed within one week after surgery. Breath-hold, axial, T2WI, multi-gradient-recalled-echo sequences were acquired (TR, $56.4 \mathrm{~ms}$; TE, 1.2-20.6 ms; flip angle, $25^{\circ}$; slice thickness, $8 \mathrm{~mm}$; matrix, $128 \times 128)$. The $\mathrm{R} 2^{*}$-parametric maps were generated with a two-parameter fitting algorithm and a confidence level of 0.01 . The $\mathrm{R} 2^{*}$ values were averaged across three ROIs of $10 \mathrm{~mm}$ in diameter. ROIs were placed on the largest cross-section of the kidney and were located peripherally within the renal parenchyma in the anterior, middle, and posterior part of the graft (Figure 1).

Measurements in transplant patients were correlated with basic laboratory parameters in the early period after transplantation, including eGFR, serum creatinine concentration ( $\mathrm{SCr}$ ), proteinuria, serum uric acid and glucose concentration, white blood cell count (WBC), red blood cell count (RBC), and haematocrit (HCT). The R2* values were also correlated with eGFR and SCr at long-term follow-up. To establish inter-reader reproducibility of measurements, $\mathrm{R} 2{ }^{\star}$ was measured on parametric maps in three cortical regions of interest (ROIs) by two observers, both with five years of experience in abdominal MRI. For intra-reader reproducibility calculation both observers repeated their measurements after six months. Images were anonymised before analysis.

Data were presented as means with $95 \%$ confidence intervals (95\% CI). Intra-reader variability and interreader variability were assessed with the coefficient of variation $(\mathrm{CV})$ and the intraclass correlation coefficient (ICC). The results from both observers were also tested for differences with repeated measures ANOVA. Standard error of measurement (SEM) was a measure of absolute agreement: SEM = SD (standard deviation) $\times$ root (1-ICC). Finally, the minimal detectable change (MDC) was calculated as the smallest change in $\mathrm{R} 2^{*}$ value that could be interpreted as a real difference [18]: $\mathrm{MDC}=1.96$ $\times$ square root of $2 \times$ SEM. Differences in oxygenation estimates between patients and controls were tested with the independent samples $t$-test. The relationship between R2* values, graft function, and laboratory parameters was analysed with multiple regression and logistic regression. $P<0.05$ was considered significant. 
Table 1. Clinical characteristics of patients

\begin{tabular}{|l|c|c|}
\hline Parameter & Mean value $(95 \% \mathrm{Cl})$ & Range \\
\hline Age (years) & $51.8(46.1-57.5)$ & $18-81$ \\
\hline $\mathrm{SCr}(\mathrm{mg} / \mathrm{dl})$ & $2.81(1.95-3.68)$ & $0.47-9.86$ \\
\hline eGFR $\left(\mathrm{ml} / \mathrm{min} / 1.73 \mathrm{~m}^{2}\right)$ & $42.5(31.3-53.6)$ & $4.8-149.1$ \\
\hline Proteinuria $(\mathrm{g} / 24 \mathrm{~h})$ & $0.55(0.37-0.73)$ & $0.00-1.00$ \\
\hline Uric acid $(\mathrm{mg} / \mathrm{dl})$ & $4.10(3.39-4.81)$ & $2.10-9.20$ \\
\hline Glucose $(\mathrm{mg} / \mathrm{dl})$ & $96.4(79.9-112.8)$ & $69.0-318.0$ \\
\hline WBC (10 $/ \mathrm{ll})$ & $8.06(7.05-9.07)$ & $3.89-16.1$ \\
\hline RBC (10 $/ \mathrm{l})$ & $3.42(3.25-3.60)$ & $2.69-5.43$ \\
\hline HCT (\%) & $31.1(29.7-32.6)$ & $25.6-47.2$ \\
\hline
\end{tabular}

\section{Results}

The clinical characteristics of patients are presented in Table 1 . We rejected examinations of seven patients (18\%) due to subjective poor image quality that was related to motion artefacts. Repeated measures ANOVA did not show significant differences between the three ROI values in either of the observers. In a per-ROI analysis, the mean intra-reader CV was 6\% for observer A and 9\% for observer B, respectively. The intraclass correlation coefficient was 0.79 and 0.87 for observer A and observer B, respectively. In a per-patient analysis, the mean inter-reader CV was $11 \%$, with a wide range of $0 \%$ to $66 \%$. The overall inter-reader CV was $14.6 \%$, SEM was $0.10 \mathrm{~Hz}$, and MDC was $0.28 \mathrm{~Hz}$.

The mean cortical R2* value was $11.6 \mathrm{~Hz}(95 \%$ CI: 11.2 to 12.0$)$ in transplant recipients and $15.9 \mathrm{~Hz}$ (95\% CI: 14.4 to 17.3$)$ in controls $(p=0.0001$, Figure 2$)$. On multiple regression analysis, we did not observe any significant relationship between the $\mathrm{R} 2{ }^{*}$ values and the studied clinical parameters. However, patients with eGFR $\geq 40 \mathrm{ml} / \mathrm{min} / 1.73 \mathrm{~m}^{2}$ had higher $\mathrm{R} 2 *$ values than those with eGFR $<40 \mathrm{ml} / \mathrm{min} / 1.73 \mathrm{~m}^{2}(12.0 \mathrm{~Hz}$ vs. $11.1 \mathrm{~Hz}$, respectively; $p=0.0189$; Figure 3 ). On ROC analysis, R2* values $\leq 11.7$ predicted impaired graft function defined as eGFR $<40 \mathrm{ml} / \mathrm{min} / 1.73 \mathrm{~m}^{2}$; sensitivity of $82 \%$, specificity of $56 \%$, area under the curve (AUC) $=0.708(p=0.024$; Figure 4).

The mean follow-up duration was 31.3 months (range: 26-45 months). Two patients were lost to follow-up. On the last measurements, the mean SCr was 1.39 (95\% CI: 1.20 to 1.58 ), and the mean eGFR was 53.1 (95\% CI: 46.3 to 59.8 ). At the long-term follow-up, $\mathrm{R} 2^{*}$ was not a significant predictor of kidney function, and there was no significant difference in post-transplant $\mathrm{R} 2{ }^{\star}$ values between patients with eGFR $\geq 40 \mathrm{ml} / \mathrm{min} / 1.73 \mathrm{~m}^{2}$ and those with eGFR $<40 \mathrm{ml} / \mathrm{min} / 1.73 \mathrm{~m}^{2}$ (11.6 vs. 11.9 , respectively). Multiple regression analysis of $\mathrm{R} 2{ }^{*}$ values

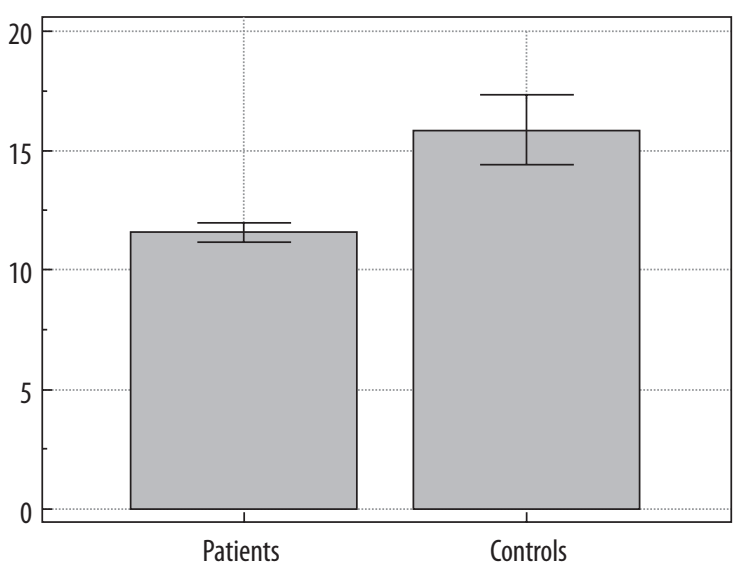

Figure 2. Comparison of cortical R2* values in transplant recipients and controls. Mean values and $95 \% \mathrm{Cls}$ are given

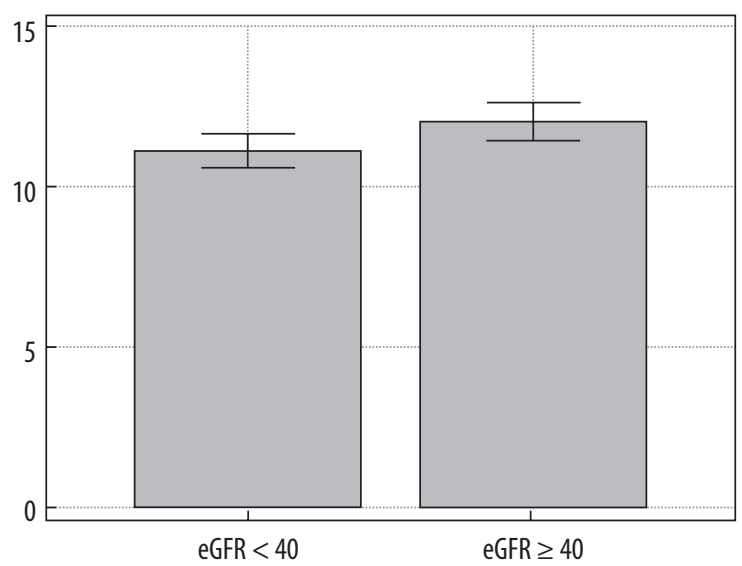

Figure 3. Comparison of cortical $R 2^{*}$ values between patients with eGFR $<40 \mathrm{ml} / \mathrm{min} / 1.73 \mathrm{~m}^{2}$ and those with eGFR $\geq 40 \mathrm{ml} / \mathrm{min} / 1.73 \mathrm{~m}^{2}$

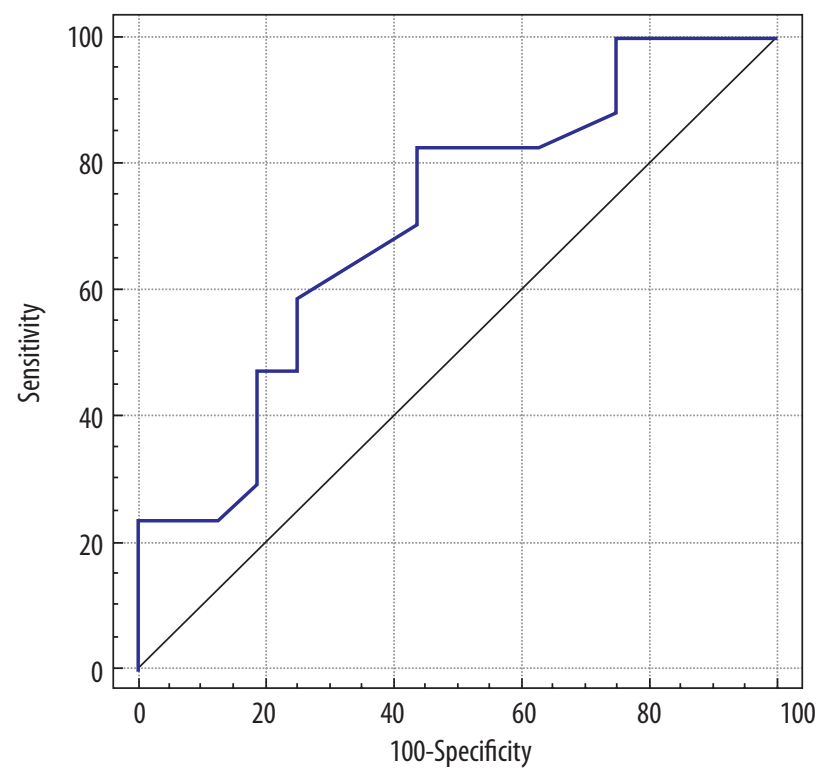

Figure 4. A ROC curve presents $R 2^{*}$ values as a predictor of an early impaired graft function defined as eGFR $<40 \mathrm{ml} / \mathrm{min} / 1.73 \mathrm{~m}^{2}$

and laboratory parameters did not allow the generation of any model to predict either early or delayed graft function. 


\section{Discussion}

To our knowledge, we showed for the first time that BOLD imaging performed early after transplantation has a limited value in predicting delayed renal graft function. We found that $\mathrm{R} 2{ }^{*}$ values were moderately related to early graft function. Finally, based on inter-reader reproducibility analysis, the minimal detectable change in $\mathrm{R} 2^{*}$ was only $0.28 \mathrm{~Hz}$.

In our study, $18 \%$ of patients were excluded from the analysis due to inadequate image quality. Similar rejection rates were reported by Sadowski et al. [14] and Niles et al. [19] but not by other researchers $[15,16,20,21]$. We suspect that this could have been due to the short time between renal transplantation and scanning. Breathing motions were the main source of image artefacts because, due to pain and common overhydration, patients in the early period after renal transplantation have a limited ability to hold their breath and to lie still in a supine position throughout the entire MRI examination. Thus, neither respiratory-gated nor breath-hold techniques guarantee images free from movement artefacts in this group of patients. However, we preferred breath-hold sequences because of shorter total examination times. Xiao et al. observed a loss of signal in the marginal area of the transplanted kidney [21] because the bowel gas surrounding the kidney caused inhomogeneity in local magnetic fields and susceptibility artefacts. We did not notice this problem in our group.

To our knowledge, renal $\mathrm{R} 2{ }^{\star}$ measurement variability has not been studied. In the current study, the intra-reader CV ranged between 6\% and 9\%, and the inter-reader variability was $14.6 \%$. In a per-patient analysis, the mean inter-reader presented a wide range of values ( $0 \%$ to $66 \%)$, which may be explained by the fact that ROI placement was left to the discretion of observers. The calculated MDC indicated that a difference of $0.28 \mathrm{~Hz}$ may be considered significant for different observers or different ROI placement methods. However, it does not take into account the physiological differences that may introduce some variability to repeated scanning. Recently, two studies have assessed the reproducibility of repeated BOLD examinations. In four patients, Park et al. reported that the mean difference in cortical or medullary $\mathrm{R} 2^{*}$ values between two MRI examinations at a one-month interval was $0.6-3.8 \%$, depending on the sequence used [20]. However, that study lacked statistical power due to a small sample. Thoeny et al., who studied only seven subjects, reported an inter-scan cortical R2 ${ }^{\star}$ variability of $4.0 \%$ [22].

Several authors have previously tested the relationship between $\mathrm{R} 2{ }^{*}$ values and graft function. Vermathen $e$ al . did not observe any relationship between eGFR and oxygenation estimates in repeated examinations performed $7 \pm 3$ months after transplantation and $32 \pm 2$ months after initial MRI [17]. Similar results were reported by Seif et al. [23]. In the study by Xiao et al., Scr levels were negatively correlated with R2* values; however, that correlation was weak (Spearman coefficient of -0.29) [21]. In our study, there was no relationship between cortical R2* values and clinical parameters, including eGFR, $\mathrm{SCr}$, and proteinuria. However, we observed a difference in renal cortical oxygenation between patients with eGFR $\geq 40 \mathrm{ml} / \mathrm{min} / 1.73 \mathrm{~m}^{2}$ and those with eGFR $<40 \mathrm{ml} / \mathrm{min} / 1.73 \mathrm{~m}^{2}$, which may indicate decreased graft function. Using this eGFR threshold, $\mathrm{R} 2{ }^{*}$ values $\leq 11.7$ predicted impaired kidney function with good sensitivity $(82 \%)$ and poor specificity $(56 \%)$. Thus, the test may be considered to rule out the graft failure but not to confirm it. Based on previous studies and our data, we can suppose that renal cortical R2*values are not strongly related to the common parameters of renal early graft function, including eGFR. Moreover, the role of this method in predicting delayed graft function is limited. We found no relationship between the $\mathrm{R} 2^{\star}$ values measured within a week after transplantation and follow-up eGFR, even if the threshold of $40 \mathrm{ml} / \mathrm{min} / 1.73 \mathrm{~m}^{2}$ was used.

Our study had some limitations. First, we included a small number of participants, but our sample size was comparable to previous studies on renal graft oxygenation. What is more, only two patients were lost for follow-up. However, the role of early R2 ${ }^{\star}$ measurement for graft function prediction should be assessed in a larger study. Since the mean follow-up duration was over 2.5 years, several factors other than the initial post-transplant renal cortex oxygenation might have influenced the final outcome. To account for such confounders, a larger study will have to be performed. Second, we only measured cortical oxygenation, which was in line with previous studies, such as the significant work by Milani et al. [11]. Finally, averaging over three ROIs might have led to some measurement errors, and averaging over whole cross-sections or even over whole cortical volumes could have been better $[11,19]$. However, such methods are still experimental and require validation before clinical application.

\section{Conclusions}

In conclusion, BOLD MRI-based evaluation of renal graft oxygenation early after transplantation is technically challenging. In practice, an $\mathrm{R} 2^{*}$ value of $0.28 \mathrm{~Hz}$ can be considered as the minimal detectable change. Despite the fact that $\mathrm{R}^{*}$ values are related to eGFR, BOLD has limited potential to predict early graft function. Delayed graft function does not seem to be dependent on early renal cortical oxygenation.

\section{Ethical approval}

All procedures performed in studies involving human participants were in accordance with the ethical standards of the institutional and/or national research committee and with the 1964 Helsinki Declaration and its later amendments or comparable ethical standards. For this type of study, formal consent is not required. 
The Institutional Review Board approved the study and did not require additional informed consent for reviewing the patients' medical records and image.

\section{Conflict of interest}

The authors report no conflict of interest.

\section{References}

1. Sharfuddin A. Renal relevant radiology: Imaging in kidney transplantation. Clin J Am Soc Nephrol 2014; 9: 416-429.

2. Thölking G, Schuette-Nuetgen K, Kentrup D, et al. Imaging-based diagnosis of acute renal allograft rejection. World J Transplant 2016; 6: 174-182.

3. Baker R, Jardine A, Andrews P. Clinical Practice Guidelines: Post-operative care of the kidney transplant recipient, UK Renal Association. 5th ed. Final version (10th December 2010).

4. Mahmoud H, Buchanan C, Francis ST, Selby NM. Imaging the kidney using magnetic resonance techniques: structure to function. Curr Opin Nephrol Hypertens 2016; 25: 487-493.

5. Gaddikeri S, Mitsumori L, Vaidya S, et al. Comparing the diagnostic accuracy of contrast-enhanced computed tomographic angiography and gadolinium-enhanced magnetic resonance angiography for the assessment of hemodynamically significant transplant renal artery stenosis. Curr Probl Diagn Radiol 2014; 43: 162-168.

6. Wang YT, Li YC, Yin LL, et al. Functional assessment of transplanted kidneys with magnetic resonance imaging. World J Radiol 2015; 7: 343-349.

7. Wypych-Klunder K, Adamowicz A, Lemanowicz A, et al. Diffusion-weighted MR imaging of transplanted kidneys: Preliminary report. Pol J Radiol 2014; 79: 94-98.

8. Robson PM, Madhuranthakam AJ, Smith MP, et al. Volumetric arterial spin-labeled perfusion imaging of the kidneys with a three-dimensional fast spin echo acquisition. Acad Radiol 2016; 23: 144-154.

9. Notohamiprodjoa M, Reiser MF, Sourbron SP. Diffusion and perfusion of the kidney. Eur J Radiol 2010; 76: 337-347.

10. Prasad PV, Edelman RR, Epstein FH. Noninvasive evaluation of intrarenal oxygenation with BOLD MRI. Circulation 1996; 94: 3271 3275.

11. Milani B, Ansaloni A, Sousa-Guimaraes S, et al. Reduction of cortical oxygenation in chronic kidney disease: evidence obtained with a new analysis method of blood oxygenation level-dependent magnetic resonance imaging. Nephrol Dial Transplant 2016; pii: gfw362.

12. Sadowski EA, Fain SB, Alford SK, et al. Assessment of acute renal transplant rejection with blood oxygen level-dependent MR imaging: initial experience. Radiology 2005; 236: 911-919.
13. Djamali A, Sadowski EA, Samaniego-Picota M, et al. Noninvasive assessment of early kidney allograft dysfunction by blood oxygen level-dependent magnetic resonance imaging. Transplantation 2006; 82: 621-628.

14. Sadowski EA, Djamali A, Wentland AL, et al. Blood oxygen leveldependent and perfusion magnetic resonance imaging: detecting differences in oxygen bioavailability and blood flow in transplanted kidneys. Magn Reson Imaging 2010; 28: 56-64.

15. Park SY, Kim CK, Park BK, et al. Assessment of early renal allograft dysfunction with blood oxygenation level-dependent MRI and diffusion-weighted imaging. Eur J Radiol 2014; 83: 2114-2121.

16. Liu G, Han F, Xiao W, et al. Detection of renal allograft rejection using blood oxygen level-dependent and diffusion weighted magnetic resonance imaging: a retrospective study. BMC Nephrol 2014; 15: 158 .

17. Vermathen P, Binser T, Boesch C, et al. Three-year follow-up of human transplanted kidneys by diffusion-weighted MRI and blood oxygenation level-dependent imaging. J Magn Reson Imaging 2012; 35: 1133-1138.

18. Haley SM, Fragala-Pinkham MA. Interpreting change scores of tests and measures used in physical therapy Phys Ther 2006; 86: 735-743.

19. Niles DJ, Artz NS, Djamali A, et al. Longitudinal assessment of renal perfusion and oxygenation in transplant donor-recipient pairs using arterial spin labeling and blood oxygen level-dependent magnetic resonance imaging. Invest Radiol 2016; 51: 113-120.

20. Park SY, Kim CK, Park BK, et al. Evaluation of transplanted kidneys using blood oxygenation level-dependent MRI at $3 \mathrm{~T}$ : a preliminary study. AJR Am J Roentgenol 2012; 198: 1108-1114.

21. Xiao W, Xu J, Wang Q, et al. Functional evaluation of transplanted kidneys in normal function and acute rejection using BOLD MR imaging. Eur J Radiol 2012; 81: 838-845.

22. Thoeny HC, Zumstein D, Simon-Zoula S, et al. Functional evaluation of transplanted kidneys with diffusion-weighted and BOLD MR imaging: initial experience. Radiology 2006; 241: 812-821.

23. Seif M, Eisenberger U, Binser T, et al. Renal blood oxygenation level-dependent imaging in longitudinal follow-up of donated and remaining kidneys. Radiology 2016; 279: 795-804. 